\title{
Using Theoretical Protein Isotopic Distributions to Parse Small-Mass-Difference Post-Translational Modifications via Mass Spectrometry
}

\author{
Timothy W. Rhoads, ${ }^{1,2}$ Jared R. Williams, ${ }^{1,2}$ Nathan I. Lopez, ${ }^{1}$ Jeffrey T. Morré, ${ }^{3}$ \\ C. Samuel Bradford, ${ }^{4}$ Joseph S. Beckman ${ }^{1,2,4}$ \\ ${ }^{1}$ Department of Biochemistry and Biophysics, Oregon State University, Corvallis, OR 97331, USA \\ ${ }^{2}$ Linus Pauling Institute, Oregon State University, Corvallis, OR 97331, USA \\ ${ }^{3}$ Department of Chemistry, Oregon State University, Corvallis, OR 97331, USA \\ ${ }^{4}$ Environmental Health Sciences Center, Oregon State University, Corvallis, OR 97331, USA
}

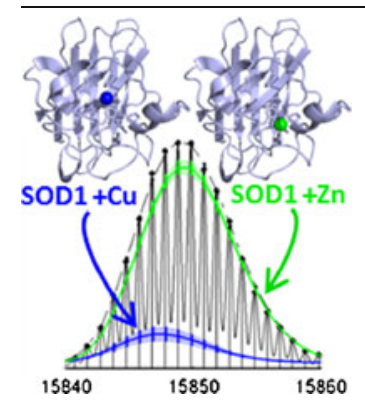

\begin{abstract}
Small-mass-difference modifications to proteins are obscured in mass spectrometry by the natural abundance of stable isotopes such as ${ }^{13} \mathrm{C}$ that broaden the isotopic distribution of an intact protein. Using a ZipTip (Millipore, Billerica, MA, USA) to remove salt from proteins in preparation for high-resolution mass spectrometry, the theoretical isotopic distribution intensities calculated from the protein's empirical formula could be fit to experimentally acquired data and used to differentiate between multiple low-mass modifications to proteins. We could readily distinguish copper from zinc bound to a single-metal superoxide dismutase (SOD1) species; copper and zinc only differ by an average mass of 1.8 Da and have overlapping stable isotope patterns. In addition, proteins could
\end{abstract} be directly modified while bound to the ZipTip. For example, washing $11 \mathrm{mM} \mathrm{S}$-methyl methanethiosulfonate over the ZipTip allowed the number of free cysteines on proteins to be detected as S-methyl adducts. Alternatively, washing with the sulfhydryl oxidant diamide could quickly reestablish disulfide bridges. Using these methods, we could resolve the relative contributions of copper and zinc binding, as well as disulfide reduction to intact SOD1 protein present from $<100 \mu \mathrm{g}$ of the lumbar spinal cord of a transgenic, SOD1 overexpressing mouse. Although techniques like ICP-MS can measure total metal in solution, this is the first method able to assess the metal-binding and sulfhydryl reduction of SOD1 at the individual subunit level and is applicable to many other proteins.

Key words: FT-MS, Superoxide dismutase, Protein isotopic distribution, Copper, Zinc, High resolution mass spectrometry

Received: 5 July 2012/Revised: 8 September 2012/Accepted: 9 September 2012/Published online: 18 December 2012

\section{Introduction}

$\mathrm{M}$ any mass spectrometers in principle have sufficient resolution to distinguish 1-2 Da mass differences in intact proteins that result from small post-translational modifications such as disulfide bond formation [1]. However, the natural abundance of ${ }^{13} \mathrm{C}$ and other stable isotopes in proteins obscures such low mass modifications because the same number of protein ions are distributed over dozens of

Electronic supplementary material The online version of this article (doi:10.1007/s13361-012-0500-1) contains supplementary material, which is available to authorized users.

Correspondence to: Joseph S. Beckman; e-mail: joe.beckman@oregonstate.edu isotopic mass peaks $[2,3]$. The intensities of the isotopic peaks can be predicted from the empirical formula of a protein with sufficient accuracy to distinguish even the addition of a single hydrogen atom [3-5]. Experimentally obtained intensities therefore contain a significant amount of information regarding the empirical formula of the molecule in question. Senko et al. used an experimental distribution based on a model amino acid, averagine, to assign the monoisotopic peak and empirical formula of myoglobin [3]. Assigning the oxidation status of a metal ion, a single Dalton mass change, has also been accomplished using isotopic distributions [6]. In this case, however, the authors only considered distributions that contained only one species. Green et al. [7] 
distinguished between two species differing by $1 \mathrm{Da}$ by identifying the tallest peak via the calculation of a centroid of the isotopic distribution over $50 \%$ of the maximum height. However, only using the top $50 \%$ of a peak ignores the smaller isotope peaks of a protein distribution that are important for distinguishing overlapping isotopic species. The challenge then is whether the isotopic distributions of proteins could be used to quantify multiple, small-mass modifications to proteins isolated from biological tissues.

Our interest stems from the need to measure the metal content of the antioxidant protein copper, zinc superoxide dismutase (SOD1), a protein genetically linked to amyotrophic lateral sclerosis (ALS), from in vivo samples. We have previously shown that a $\mathrm{C}_{4}$-reversed phase ZipTip could be used to measure SOD1 from less than $100 \mu \mathrm{g}$ of spinal cord from transgenic rats [8]. The ZipTip allowed salts and other contaminants that would otherwise interfere with mass spectrometry to be removed by washing with water. After washing, the ZipTip was mounted directly in-line with the solvent line leading to the electrospray interface of a Time-of-Flight (ToF) mass spectrometer and proteins were eluted with a low percentage of acetonitrile.

Although conditions for electrospray ionization could be made sufficiently gentle with the ZipTip method to keep metals bound to SOD1, a majority of the protein isolated from the spinal cord of transgenic animals was missing one metal [8]. The missing metal from SOD1 in these tissues could be either copper or zinc, whose average masses differ by only 1.8 Daltons. Our method using ToF mass spectrometry lacked the resolution to distinguish such overlapping species. Determining whether the remaining metal was copper or zinc in vivo is important because we have previously shown that copper-containing, zinc-deficient SOD1 is toxic to motor neurons [9], but have no means of assaying zincdeficient SOD1 from transgenic animal model tissue.

The measurement of zinc-deficient SOD1 is further complicated because the protein has an unusually stable intrasubunit disulfide bond restraining the zinc-binding loop. Additionally, the disulfide is known to be partially reduced in transgenic SOD1 overexpressing animals [10-15]. The extent of disulfide reduction in vivo is controversial because of differences in measurement methods between laboratories.

No method has been able to simultaneously determine which metals remain bound to disulfide-reduced versus oxidized SOD1 subunits in vivo. To address these issues, we adapted the ZipTip-based assay for use with a Fourier transform ion cyclotron resonance (FT-ICR) mass spectrometer to accurately measure the isotopic intensity distributions of intact proteins. By matching the masses of the predicted isotope distribution calculated from SOD1's empirical formula to the experimental high-resolution mass spectrum, the intensities could distinguish the contributions that each metal ion makes to the experimental isotopic distribution of SOD1. Reduction of the disulfide bond was independently assessed by modifying reduced cysteines with S-methyl methanethiosulfonate (MMTS). The combination of these methods should be useful for probing multiple modifications to other proteins in addition to SOD1.

\section{Methods}

\section{Mass Spectrometry Methods}

Binding of recombinant or tissue-derived proteins to a ZipTip was an efficient and disposable means for removing salts and introducing proteins into an electrospray mass spectrometer [8]. In brief, a C4 ZipTip was prepared first by rinsing three times with acetonitrile and four times with water $(10 \mu \mathrm{L}$ each). Proteins were then adsorbed onto the tip by repeatedly drawing the sample solution through the ZipTip eight times with a pippetter, followed by six washes with water to remove salts. The ZipTip was directly inserted in-line between an isocratic HPLC pump and a microfilter leading to the electrospray source as previously described. Elution of SOD1 from the ZipTip is nearly complete after three minutes and successive scans over this interval are averaged together.

Proteins were detected using a LTQ-FT Ultra hybrid linear ion trap-Fourier transform ion cyclotron resonance mass spectrometer (Thermo, San Jose, CA, USA) with a Finnigan Ion Max API source configured for electrospray ionization (ESI) interface in positive ion mode. Measurements to determine the specific metals bound to the protein were accomplished in the ICR cell at a chosen resolution of 100,000 defined at $400 \mathrm{~m} / \mathrm{z}$. Quantitation of total protein levels using bovine SOD1 as an internal standard, similar to the previous study [8], used the linear ion trap (LTQ) mode.

The solvent used for the mass spectrometric experiments was $30 \%$ acetonitrile, $70 \%$ water and $100 \mu \mathrm{M}$ formic acid, delivered at a flow rate of $20 \mu \mathrm{L} / \mathrm{min}$ from a single isocratic pump (Shimadzu, Kyoto, Japan). The solvent was degassed for 10 minutes with helium and was changed each week. HPLC-grade solvents were used for all mass spectrometer experiments. Water was obtained from Burdick and Jackson (Muskegon, MI, USA).

\section{Protein Preparation}

All chemicals were obtained from VWR unless otherwise noted. Chicken lysozyme, bovine ubiquitin, and MMTS were obtained from Sigma-Aldrich (St. Louis, MO, USA). Bovine SOD1 from erythrocytes was obtained from Sigma. Its concentration was determined spectrophotometrically at $258 \mathrm{~nm}\left(\varepsilon=5150 \mathrm{M}^{-1} \mathrm{~cm}^{-1}\right.$ for the Cu,Zn SOD1 monomer). All SOD1 concentrations refer to the monomer concentration rather than dimer. Wild type and pseudo-wild type C111S human SOD1 expressed in Escherichia coli (hence referred to as recombinant SOD1) with coexpression of the copper chaperone for SOD1 (CCS) were used for developing many of the assays. C111S SOD1 is more stable than wildtype human SOD1 and was used here to verify the rapid modification of a single thiol was due to C111. The SOD1 protein was purified as described in Hayward et al. [16], and the concentration was determined spectrophotometrically at $265 \mathrm{~nm} \quad\left(\varepsilon=9200 \mathrm{M}^{-1} \mathrm{~cm}^{-1}\right.$ for the $\mathrm{Cu}, \mathrm{Zn}$ containing 
monomer) [17]. Recombinant forms of human SOD1 were not acetylated on the N-terminus.

To generate copper- or zinc-containing single metal SOD1 standards, both metals were stripped from $\mathrm{Cu}, \mathrm{Zn}$ SOD1 by incubating the protein $(>1 \mathrm{mg} / \mathrm{mL})$ in $50 \mathrm{mM}$ acetate $\mathrm{pH} 3.7$ with $1.5 \mathrm{mM}$ EDTA. Once all metals were removed, copper-containing SOD1 was prepared by making multiple additions of $15 \mu \mathrm{M}$ copper nitrate (atomic absorption standard, Sigma-Aldrich) in $10 \mathrm{mM}$ potassium acetate at $\mathrm{pH} 4.15$ plus $10 \mathrm{mM}$ sodium chloride to the SOD1 in an Amicon Ultra $0.5 \mathrm{~mL}$ centrifugal filter with a $3 \mathrm{k}$ MWCO. This was centrifuged for 10 minutes at 16,000 rcf after each addition. Zinc-containing SOD1 was prepared in a similar manner through multiple additions of $15 \mu \mathrm{M}$ zinc chloride atomic absorption standard (Sigma-Aldrich), in $10 \mathrm{mM}$ potassium acetate at $\mathrm{pH} 4.6$ with $10 \mathrm{mM}$ sodium chloride. The number of metal additions was determined by monitoring metal incorporation into SOD1 after each addition via mass spectrometry. All buffer solutions and water were run through a Chelex 100 (50-100 dry mesh, Sigma) column prior to use.

SOD1 was also measured from tissue samples of wildtype SOD1-overexpressing transgenic mice (B6SJL-Tg (SOD1)2Gur/J). In eukaryotic sources, SOD1 is acetylated on the amino-terminus, increasing the protein mass by 42.1 Da. All animal procedures and housing arrangements were approved by the Institutional Animal Care and Use Committee. Animals sacrificed for tissue harvest were placed under isofluorane anesthesia followed by pericardial perfusion with heparinized PBS and decapitation. Fresh spinal cord tissues from transgenic WT-SOD1 mice were extracted via dissection of euthanized animals and frozen at $-80{ }^{\circ} \mathrm{C}$ until use. Frozen necropsy punches taken from $1 \mathrm{~mm}$ thick spinal cord slices were thawed in $10 \mu \mathrm{l}$ of $10 \mathrm{mM}$ ammonium acetate, adjusted to $\mathrm{pH} 5.1$ with acetic acid. The punch was centrifuged and the supernatant loaded on to a ZipTip.

Table 1. Instrumental Parameters for LTQ-FT Ultra Mass Spectrometer

\begin{tabular}{ll}
\hline ESI source & \\
Sheath gas & 2 arb units \\
Auxillary gas & 1 arb units \\
Sweep gas & 1 arb units \\
Spray voltage & $5 \mathrm{kV}$ \\
Capillary temp. & $200{ }^{\circ} \mathrm{C}$ \\
Capillary voltage & $36 \mathrm{~V}$ \\
Tube lens & $205 \mathrm{~V}$ \\
Scan parameters & \\
Mass range & $800-2000 \mathrm{~m} / z$ \\
Resolution & 100000 \\
Scan type & Full \\
Microscans & 1 \\
Max. inject time & $8000 \mathrm{~ms}$ \\
ICR cell parameters & \\
Trapping voltage & Pos $0.51 \mathrm{Neg}-0.40$ \\
Excite amplitude & fm $>190(+): 0.64$ \\
& fm $>98(+): 0.5$ \\
Injection control & fm $>50(+): 0.75$ \\
Full MS & \\
\end{tabular}

\section{Thiol Reduction Assays}

Probing the oxidation status of free cysteines was done using MMTS, while the thiol oxidant diamide was used to ensure all disulfide bonds were oxidized. This served as a control for the experiments using MMTS to ensure all protein disulfide bonds were fully oxidized. MMTS modifies free thiols with a methylthiol group. Proteins were first adsorbed onto the ZipTip as above, rinsed once with $10 \mu \mathrm{L}$ of $11 \mathrm{mM}$ MMTS (a 1000-fold dilution of liquid MMTS) and then six times with distilled water. This short exposure time $(<5 \mathrm{~s})$ was sufficient to modify all free thiols in SOD1. In developing the MMTS assay for other proteins, internal disulfides in lysozyme were reduced by preincubation with $100 \mu \mathrm{M}$ tris(2-carboxyethyl)phosphine (TCEP).

The ratio of ${ }^{13} \mathrm{C}$ incorporated into a protein can vary depending on the source of carbon in either the bacterial media for recombinant proteins or in the mouse diet for tissue samples,
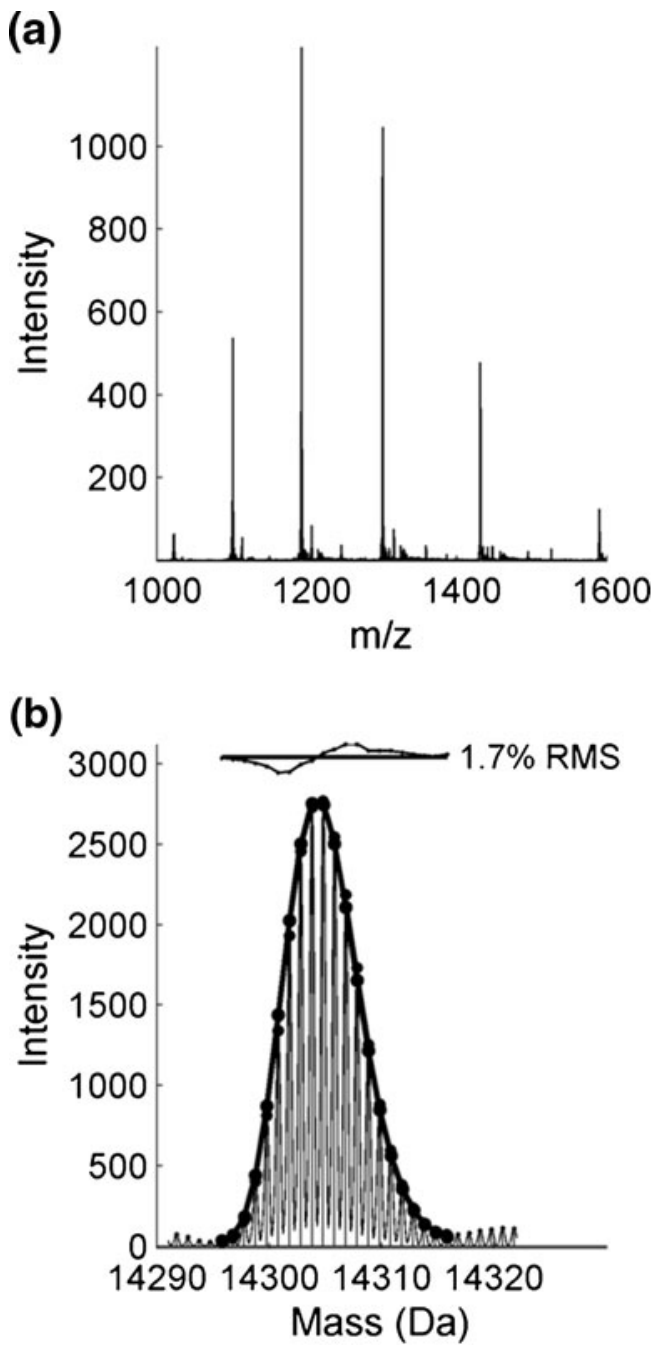

Figure 1. Mass spectra of chicken lysozyme. (a) The $\mathrm{m} / \mathrm{z}$ distribution of chicken lysozyme eluted from the ZipTip, showing charge states 9-14. (b) The theoretical isotope distribution of chicken lysozyme, based on the empirical formula $\mathrm{C}_{613} \mathrm{H}_{951} \mathrm{~N}_{193} \mathrm{O}_{185} \mathrm{~S}_{10}$, was fit to the parent mass spectrum from the experimental distribution shown in (a) 
which in turn reflects differences in carbon fixation between $\mathrm{C} 3$ and C4 plants [18]. Stable isotope analysis of tissues and purchased proteins was carried out by the Stable Isotope Research Unit (SIRU), Department of Crop and Soil Sciences, Oregon State University. Samples were analyzed for total carbon and ${ }^{13} \mathrm{C}$ on a PDZ Europa 20-20 isotope ratio mass spectrometer interfaced with a SerCon GSL elemental analyzer (SerCon, Crewe, UK). To determine \% carbon, dry sample material was weighed to the nearest microgram on a Cahn microbalance prior to combustion. Liquid samples were pipetted onto an inert sorbant material (Chromosorb; SerCon, Crewe, UK). Samples were packaged in pressed tin capsules (Elemental Microanalysis, Devon, UK). The laboratory-working standard was NIST SRM1547 peach leaves $\left(45.54 \% \mathrm{C}, \delta{ }^{13} \mathrm{C}\right.$ VPDB
-25.99). To correct for possible drift in the isotope ratio mass spectrometry (EA/IRMS) combination with time, drift correction standards of equal mass were run every 10 samples. Additional standards were run as well after every 10 samples. Stable isotope ratios were reported in delta notation as parts per thousand deviation from the international standard V-PDB. Typical precision for the SIRU 20-20 IRMS was $<0.2$ parts per thousand deviation for ${ }^{13} \mathrm{C}$. These values were incorporated into the theoretical protein isotopic distribution calculations.

\section{Data Analysis}

Spectra were collected using Tune-Plus (Thermo, ver. 2.2) page of Xcalibur (Thermo, ver. 2.0.5) using the parameters

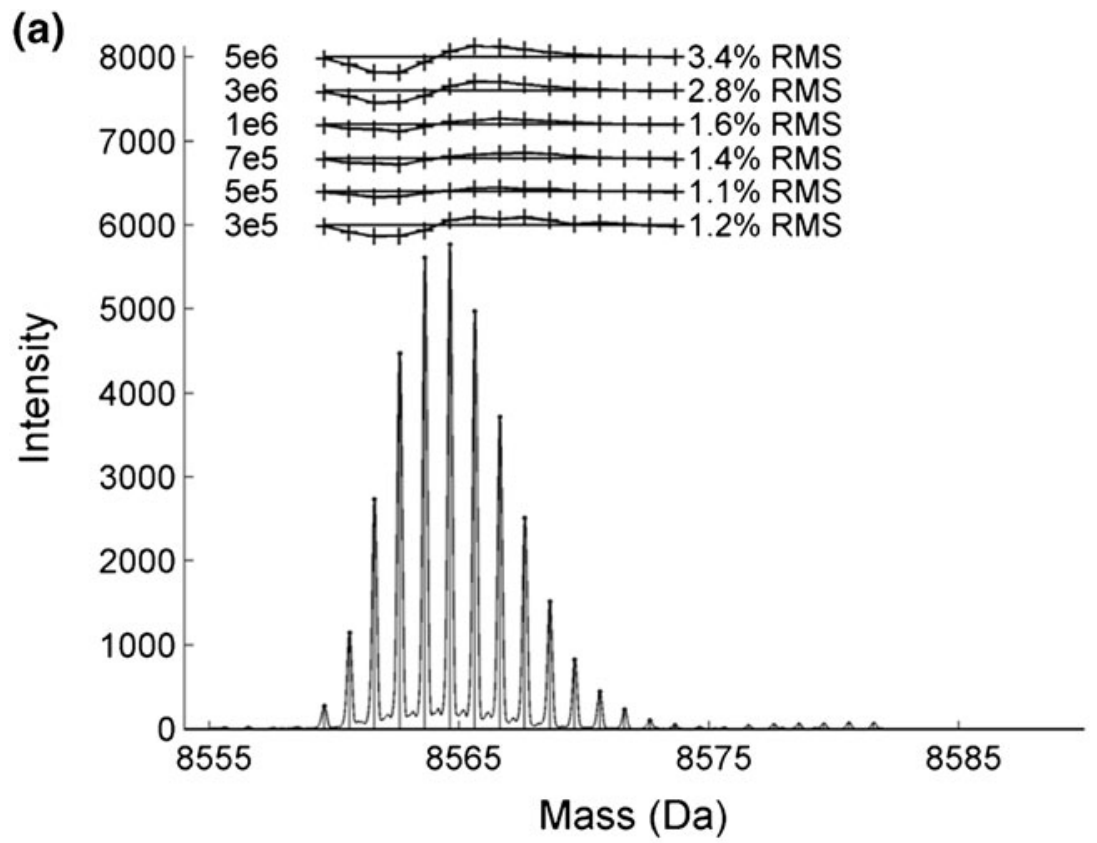

(b)

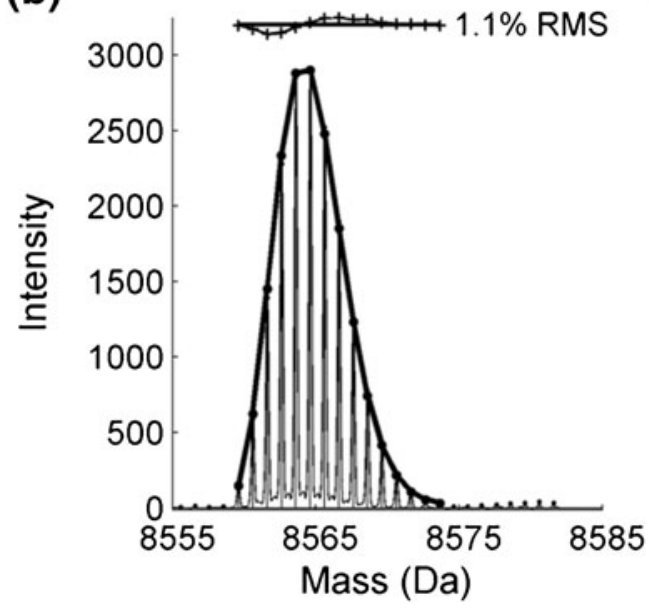

(c)

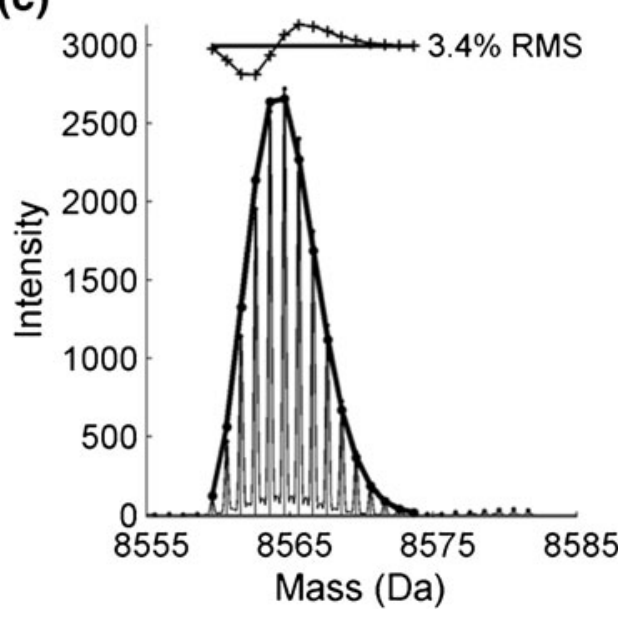

Figure 2. Effect of ion cell fill on the isotopic distribution of ubiquitin. (a) Bovine ubiquitin was fit with the program at ICR cell AGC settings from $3 \mathrm{e} 5$ to $5 \mathrm{e} 6$, demonstrating a systematic deviation from the expected distribution at ion fill levels below and above 5e5. (b) Ubiquitin fit at the optimal 5 e5 ion fill. (c) Ubiquitin fit at $5 \mathrm{e} 6$ ion fill, showing the increased systematic deviation of residuals with overfilling 
given in Table 1. In addition, the FTICR cell trapping potentials were also optimized during a continuous infusion of bovine SOD1 to further improve the theoretical fits to experimental isotopic distributions. Spectra were averaged over the three minutes that SOD1 eluted from the ZipTip using the Qual Browser (Thermo, ver. 2.0). The data were exported as text files containing two columns of $\mathrm{m} / \mathrm{z}$ versus intensity to be analyzed by custom programs written in MatLab (available in Supplementary Materials). The baseline was subtracted and centroids calculated from the experimental data using programs provided in the Bioinformatics Toolbox of MatLab. The parent mass distribution of SOD1 was reconstructed by summing the spectral regions containing individual charge states of SOD1 multiplied by their charge after subtracting the number of hydrogen atoms corresponding to the charge state [19]. Bayesian deconvolution of the mass/charge spectra as previously used with our ToF measurements [8] was not necessary due to the clarity and high signal/noise ratio of the spectra.

Intensities of theoretical isotopic distributions were calculated for apo, copper-containing, zinc-containing and $\mathrm{Cu}, \mathrm{Zn}$ SOD1 via the Rockwood algorithm $[4,5]$ using only the empirical formulas of the particular SOD1 mutant protein under investigation and the experimentally determined ${ }^{13} \mathrm{C} /{ }^{12} \mathrm{C}$ ratios. The theoretical isotopic distributions calculated for SOD1 were calculated for electrically neutral parent molecules, so a hydrogen ion was removed for each charge carried by the metal ions bound to SOD1. Two hydrogen atoms were subtracted for the reduction of the intra-molecular disulfide bond between C57 and C146. The theoretical spectra also were calculated with multiple additions when SOD1 was treated with the sulfhydrylmodifying agent MMTS.

Fitting of experimental to theoretical data was done in two steps. The predicted $\mathrm{m} / \mathrm{z}$ centroids from the theoretical isotopic spectra were first matched to the closest $\mathrm{m} / \mathrm{z}$ values for experimental centroid peaks for each charge state of SOD1 in the experimental spectra. The Levenberg-Marquardt fitting algorithm was then used to determine the optimal scaling of the normalized intensities of theoretical spectra with overlapping distributions to match experimentally measured centroid data. The fit was constrained to allow only positive intensities. Predicted isotope intensities less than $1 \%$ were ignored in the fitting program. The scaling factors were summed over all observable charge states to determine the relative contribution of each species to the overall observed spectrum.

For clarity, the fits are shown as parent masses rather than as individual $\mathrm{m} / \mathrm{z}$ peaks. The program also generates the fits for each $\mathrm{m} / \mathrm{z}$ as well as the charge state distributions.

\section{Results and Discussion}

The ZipTip offers a simple, fast, and reliable method to isolate proteins in complex samples sufficiently free of interference to allow accurate measurement of the isotopic (a)
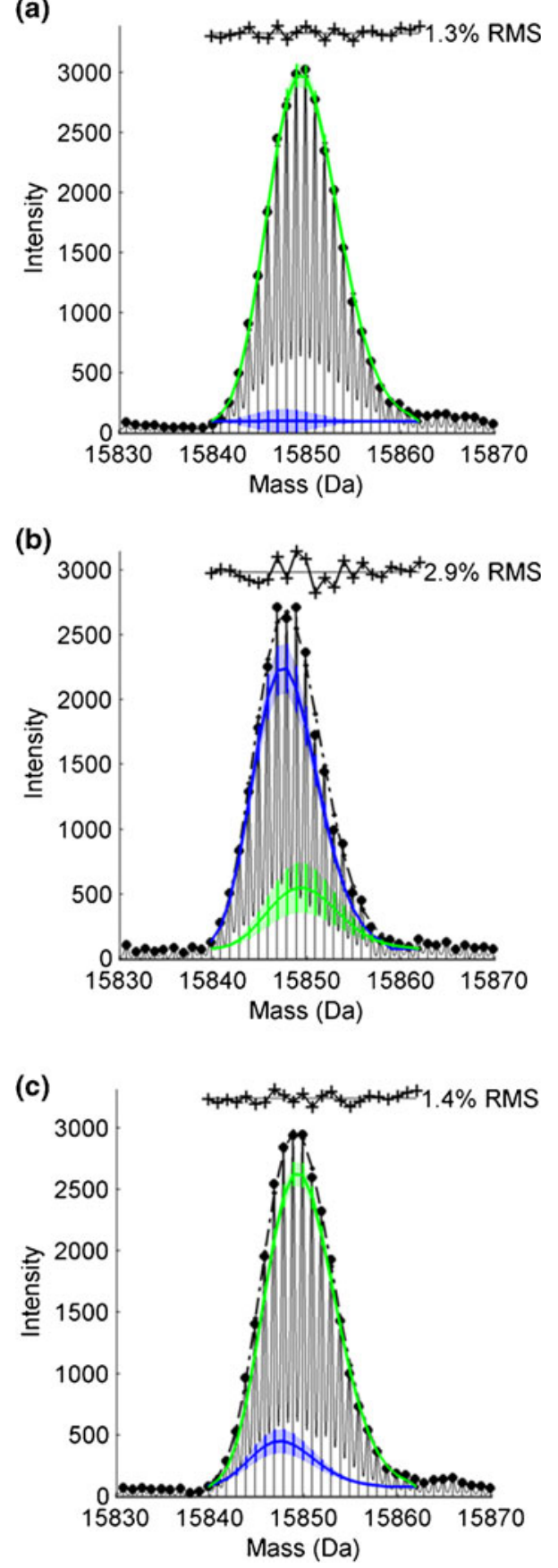

Figure 3. C111S SOD1 single metal preparations. (a) C111S mutant SOD1 single-metal standard was prepared with only zinc (green fit) as described in the methods. (b) C111S mutant SOD1 single-metal standard was prepared with only copper (blue fit) as described in the methods. A small amount of residual zinc (green fit) was consistently present that we were unable to remove. (c) C111S mutant SOD1 single-metal standard prepared with only zinc (green) or only copper (blue) and then mixed to produce a $100 \%$ mixture of $10 \%$ zincdeficient and $90 \%$ copper-deficient SOD1. The resulting fit to the theoretical isotope distributions found $12 \% \pm 2.9 \%$ zinc-deficient SOD. Confidence intervals (95\%) are displayed as a colored, transparent range around the best fit line for each species 
distribution (Figure 1a). As illustrated with lysozyme, the isotopic intensities predicted from the empirical formula of lysozyme with four disulfide bonds accurately fit to the experimentally acquired isotope distribution (Figure 1b). The experimental data for several small proteins could be fit with a root-mean square of $1 \%-2 \%$. Such accuracy allowed low mass post-translational protein modifications to be resolved.

Controlling the number of ions in the FTICR proved to be important for accurately resolving the isotopic distribution and the ion fill needs optimization for each type of protein. Increasing the ion fill beyond an empirically determined limit distorted the isotopic distribution as seen with ubiquitin
(Figure 2a-c). The upper limit likely results from space charge repulsion between protein ions that affects the course of the free-induction decay used to measure the mass to charge of protein ions [20, 21]. Overfilling of the ion cell causes the central isotopic peaks of the protein's distribution to become magnified, while the smaller peripheral peaks were deemphasized [22]. These deviations can be identified from a residual plot of the deviations (Figure 2c). At lower ion fill levels, sensitivity diminished and the decreased signal-to-noise resulted in worse fitting to the predicted spectra. Consequently, the ion fill needed to be experimentally optimized for each type of protein, with smaller proteins generally fitting better with lower ion fills.
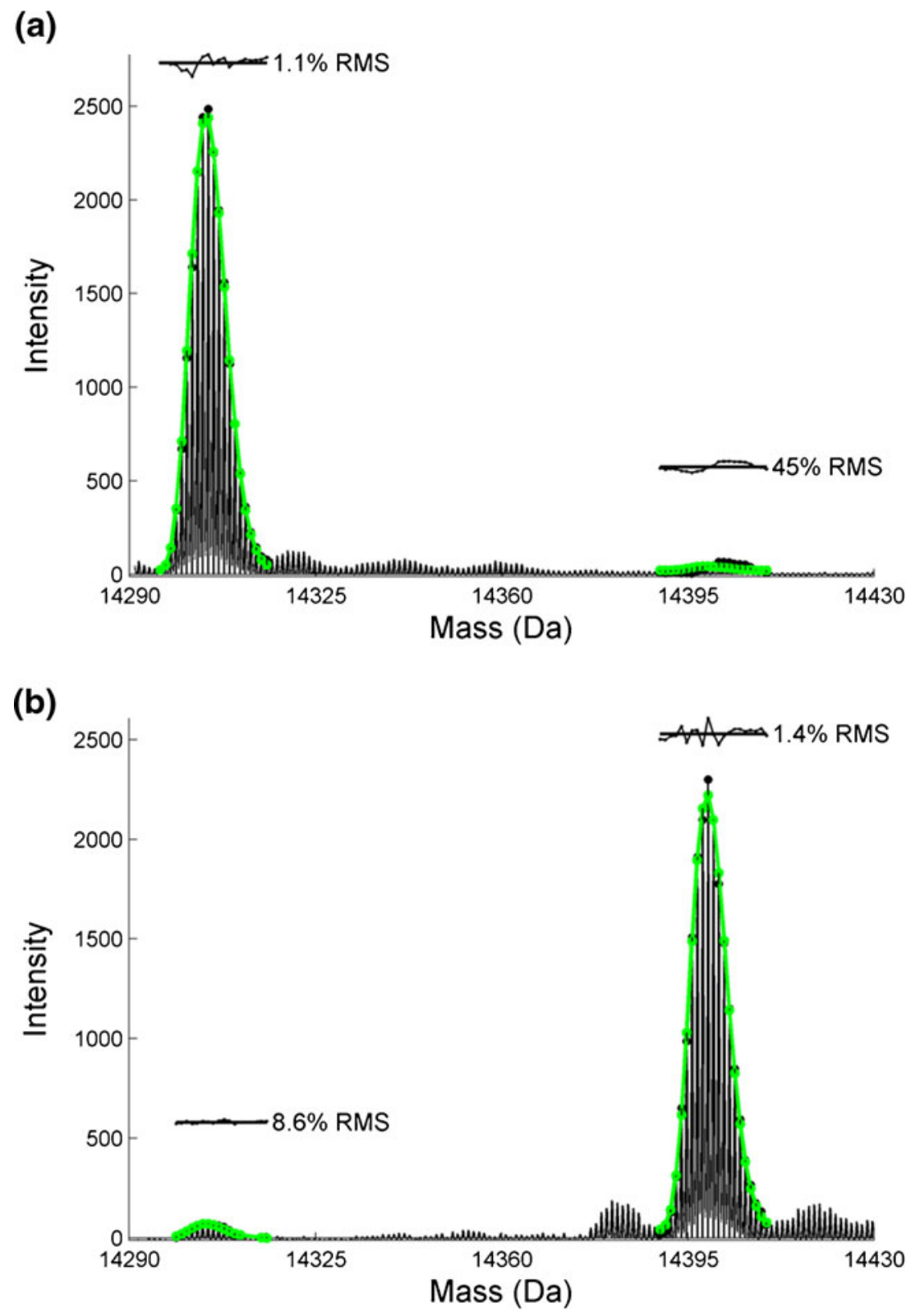

Figure 4. Use of MMTS to determine thiol oxidation status of lysozyme. Lysozyme was subjected to MMTS treatment on the ZipTip as described in the methods after incubation with TCEP for (a) zero min, showing no modifications, or (b) 30 min, demonstrating two methylthiol additions, consistent with complete reduction of one disulfide bond 
In developing the Matlab programs to fit predicted isotopic distributions to experimental data, we customized the Rockwood algorithm [4, 5] for calculating isotopic intensity distributions to allow different abundances of isotopes to be included. The largest contribution to the isotopic distribution is the ratio of ${ }^{13} \mathrm{C}$ to ${ }^{12} \mathrm{C}$ incorporated into proteins, which can vary significantly in nature [18]. The major source of this variation results from different photosynthetic processes used to fix carbon dioxide. Due to compartmentalization of different steps of carbon fixation, plants such as corn that use the $\mathrm{C} 4$ pathway discriminate against the incorporation of ${ }^{13} \mathrm{C}$ less than plants relying entirely on the C3 Calvin cycle [23]. Proteins from animals fed diets enriched in corn can, therefore, have a significantly greater amount of ${ }^{13} \mathrm{C}$ incorporated, which can be observed in the spectra $[24,25]$. These ratios can be independently measured by inductively coupled plasma mass spectrometry and incorporated in the calculation of the predicted spectra. The overall effect resulted in relatively small corrections of $<1 \%$ in the RMS for the fitted plots.

The isotopic distributions of copper and zinc add considerable complexity to the spectrum of SOD1 and are challenging to resolve because copper and zinc are neighbors on the periodic chart. Yet distinguishing how much copper can be lost compared to zinc from SOD1 in vivo is crucial for understanding how this enzyme malfunctions to cause motor neuron death in ALS. For the metal-binding SOD1 proteins, one hydrogen ion was subtracted from the

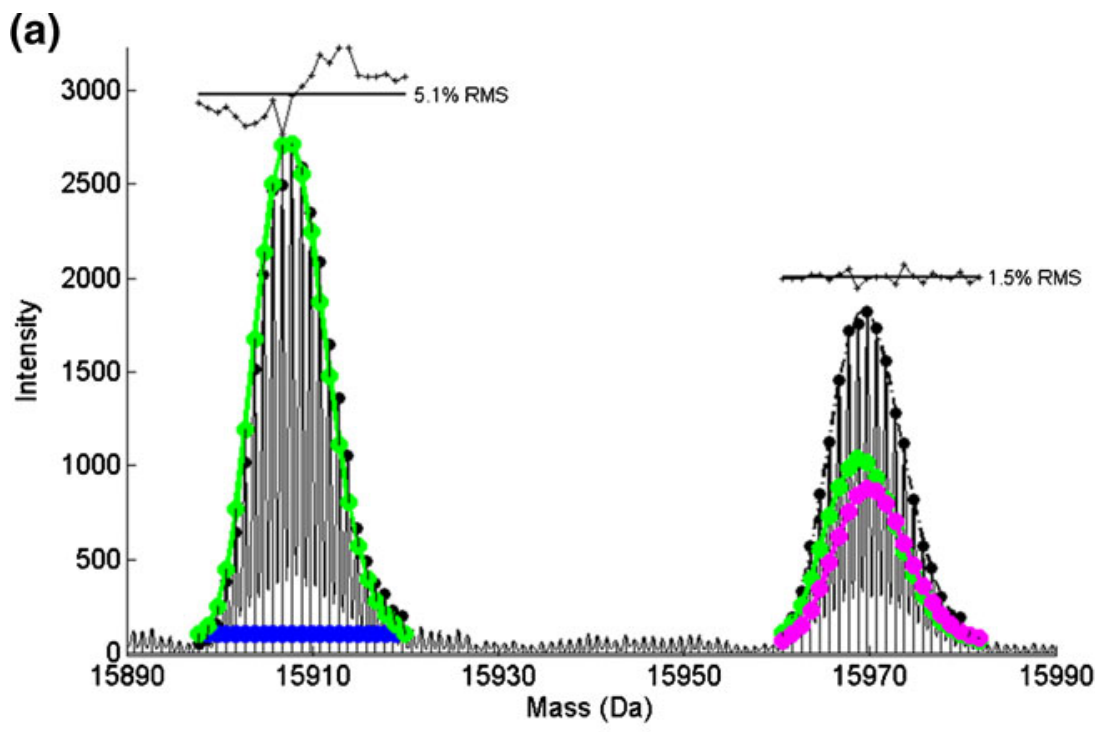

(b)

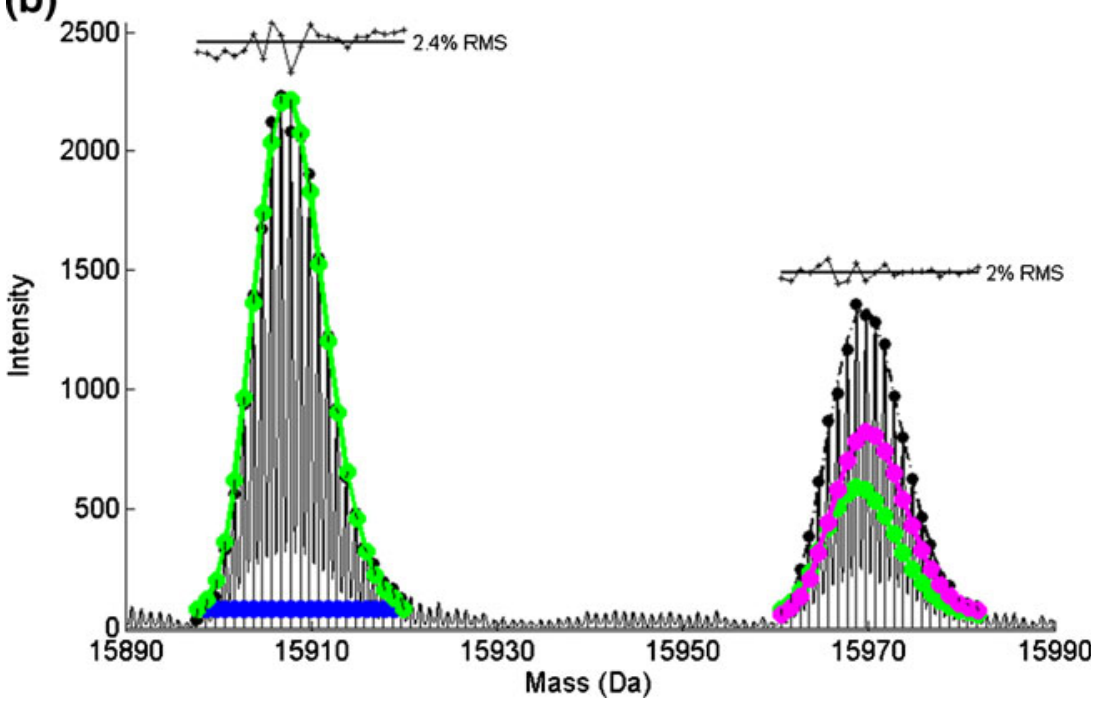

Figure 5. SOD1 isolated from the ventral spinal cord of a WT transgenic mouse with and without diamide. A ventral spinal cord punch was analyzed via the ZipTip assay as described in methods. (a) The one-metal SOD1 peak without diamide shows a systematic deviation in the residuals indicative of the presence of a higher mass species, as would be expected for SOD1 with a reduced disulfide bond. (b) The same tissue treated with diamide present fit zinc-containing SOD1, indicating complete oxidation of the disulfide bond. The amount of disulfide reduction was confirmed with the MMTS assay 
empirical formula to account for each charge on each metal ion. Copper-deficient and zinc-deficient SOD1 standards were used to validate the fitting program's ability to differentiate between copper and zinc in a one-metal peak (Figure $3 \mathrm{a}-\mathrm{c}$ ). C111S SOD1 prepared with only zinc fit as almost exclusively zinc-containing SOD1 (Figure 3a). C111S SOD1 prepared with only copper fit as mostly copper-containing SOD1 with a small amount of residual zinc-containing SOD1 (Figure 3b).

These two single-metal standards, when combined to defined concentrations of $100 \mathrm{nM}$ copper-containing SOD1 and $900 \mathrm{nM}$ zinc-containing SOD1, produced an isotopic distribution that was fit containing $12 \%$ copper $( \pm 2.9 \%)$ and $88 \%( \pm 3.2 \%)$ zinc (Figure $3 \mathrm{c})$. However, an additional potential confounding factor is the reduction of copper from $\mathrm{Cu}^{2+}$ to $\mathrm{Cu}^{1+}$ that is the key event in the standard catalytic cycle of SOD1. Reduction of iron by one electron has been observed for iron-containing heme proteins in the gas phase [26]. Copper reduction will increase the apparent mass of SOD1 containing the two stable ${ }^{63} \mathrm{Cu}$ and ${ }^{65} \mathrm{Cu}$ isotopes to equal the mass of SOD1 containing the ${ }^{64} \mathrm{Zn}$ isotope (48 \% abundance) or ${ }^{66} \mathrm{Zn}$ isotope ( $28 \%$ abundance). Hence, the deconvoluted spectrum of reduced zinc-deficient copper-containing SOD1 will significantly overlap with that of SOD1s containing the two predominant zinc isotopes and will underestimate the amount of copper-containing SOD1. Fortunately, this issue appears to be minor in practice because reduction of copper in purified zinc-deficient SOD1 during electrospray ionization was not observed in any of our zinc-deficient SOD1 standards. Experiments with chemically reduced, purified zinc-deficient SOD1 also appeared to be completely oxidized in the mass spectrometer. This is consistent with copper being more exposed in zincdeficient SOD and susceptible to being oxidized during the electrospray ionization process.

The copper in $\mathrm{Cu}, \mathrm{Zn}$ SOD1 is far less accessible and in contrast to zinc-deficient SOD1 becomes partially reduced during ionization. The observed spectrum for $\mathrm{Cu}, \mathrm{Zn}$ SOD1 could only be reasonably fit by assuming a fraction of the copper was reduced. These fits typically found that $20 \%-$ $50 \%$ of $\mathrm{Cu}, \mathrm{Zn}$ SOD1 was reduced. The reduction happened during the ionization process, because it was observed with both purified bovine and human SOD1 that were fully oxidized. These purified SOD1s were confirmed to be fully oxidized before introduction into the mass spectrometer from measuring the cuprous $\mathrm{d}-\mathrm{d}^{*}$ band, which maximally absorbs at $680 \mathrm{~nm}$. Reduced SOD1 has no absorbance at this wavelength. Chemically reduced purified $\mathrm{Cu}, \mathrm{Zn}$ SOD1 also appeared to be a mixture of reduced and oxidized SOD. These results might be explained by the oxidation of formate within the steel electrospray needle to formate radical, which in turn will reduce oxygen to superoxide. The superoxide produced would result in a rapid reduction of oxidized SOD1 as well as oxidation of reduced SOD1 to produce the mixture of reduced and oxidized $\mathrm{Cu}, \mathrm{Zn}$ SOD1 observed.

A further challenge to resolve is the potential reduction of the disulfide bond of SOD1, which is known to occur in
SOD1 transgenic mice [11]. When the intramolecular disulfide bond of SOD1 is reduced the protein mass is increased by $2 \mathrm{Da}$, complicating the measurement of copper versus zinc bound to SOD1. We found that disulfide bonds could be quickly reoxidized by a brief treatment with the thiol oxidant diamide. With the ambiguity of disulfide reduction eliminated, the fitting program could easily parse zinc from copper bound to SOD1.

(a)

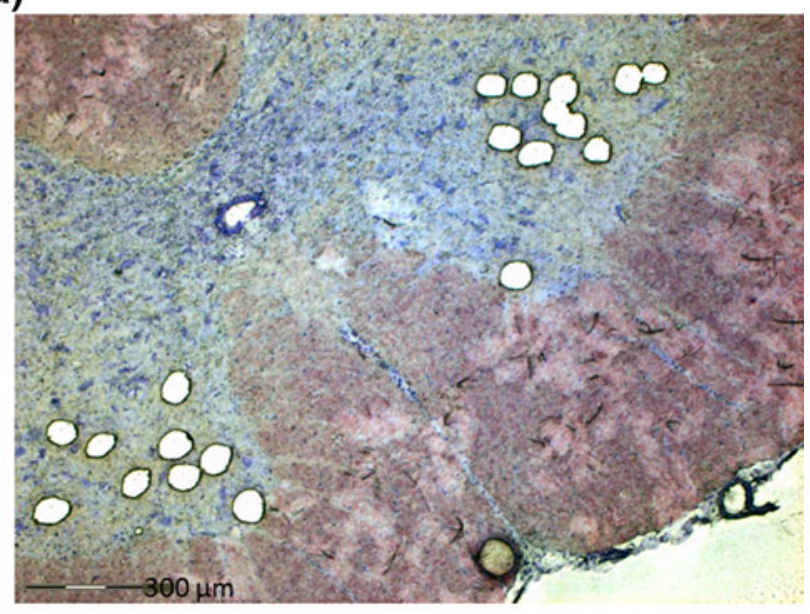

(b)

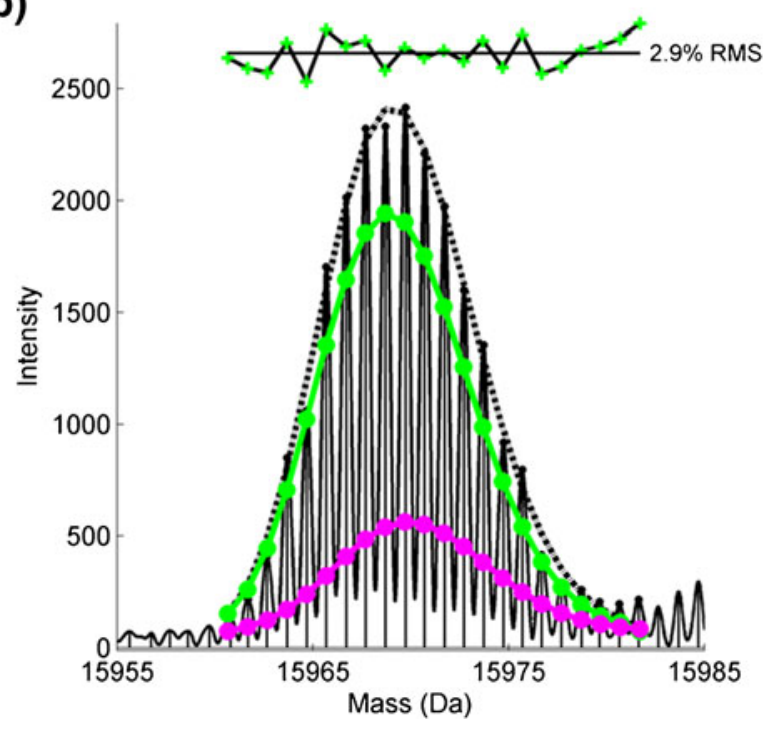

Figure 6. SOD1 isolated from 400 motor neurons captured via laser capture microdissection. The spinal cord from a WT transgenic rat was frozen and embedded in O.C.T. (Optimal Cutting Temperature) sectioning medium. Twenty $\mu \mathrm{m}$-thick sections were cut using a cryostat and then stained with Cresyl violet. (a) A Zeiss Laser Capture microdissection instrument was used to cut and capture approximately 400 motor neurons from eight sections into $20 \mu \mathrm{L}$ of $10 \mathrm{mM}$ ammonium acetate. A 50x magnification of one section is shown. Scale bar is $300 \mu \mathrm{m}$. (b) A mass spectrum of the capture buffer was acquired using a ZipTip, showing $\mathrm{Cu}, \mathrm{Zn}$ SOD1 from the motor neurons 
A separate assay was developed to directly assess whether disulfide bonds were reduced by using thiol modifying agents to react with cysteine. The majority of thiol modifying agents, including iodoacetamide, iodoacetic acid, vinyl pyridine, and several maleimides, reacted too slowly to be useful in the present assay. In addition, many modifiers introduced adducts whose mass overlapped with other metal-binding states of SOD1. We found that MMTS, in contrast, was particularly well suited to modify free thiols of proteins bound on the ZipTip. Each MMTS added $-\mathrm{SCH}_{3}$ $(47.1 \mathrm{Da})$ to each free thiol within seconds of exposure. Hence, modification of the reduced disulfide bond increased a protein mass by $94.2 \mathrm{Da}$ relative to those with an oxidized disulfide bond, allowing disulfide-reduced proteins to be clearly resolved from those with intact disulfide bridges.

Lysozyme contains four disulfide bonds and does not bind metals like SOD1, making it a convenient molecule for perturbing disulfide bond status and validating MMTS for use as a probe for free thiols. Using TCEP to reduce disulfides in lysozyme, MMTS was utilized to monitor the formation of free thiols over time (Figure 4). A $100 \mathrm{mM}$ concentration of TCEP was sufficient to fully reduce one disulfide bond (Figure $4 \mathrm{~b}$ ) and partially reduce a second, leading to a majority of the protein modified with 2 MMTS molecules and a small population modified with 4 MMTS.

When treated with MMTS, purified SOD1 bound one methyl thiol group immediately, which was most likely due to modification of cysteine residue 111. The modification did not occur using the pseudo-wild type SOD1 containing the mutation of cysteine 111 to serine. One caveat with MMTS is that the methyl thiol adduct is the intermediate formation of a mixed disulfide and might exchange with a neighboring cysteine to reoxidize the disulfide bridge [27]. This was minimized by using a high concentration of MMTS $(11 \mathrm{mM})$ to modify all free thiols within seconds.

The diamide and MMTS assays were used to measure disulfide reduction in human SOD1 measured from a $100 \mu \mathrm{g}$ punch of SOD1-overexpressing mouse spinal cord. The measurement from tissues was previously described using tissue from transgenic rats [8]. In mouse, the one-metal SOD1 comprised almost $60 \%$ of total SOD1 while roughly $35 \%$ of the SOD1 contained both copper and zinc (Figure 5). Apo SOD1 represented less than $5 \%$ of the total SOD1. The one-metal peak fit entirely as zinc-containing SOD1 with no copper present. Without diamide present, the one-metal SOD1 peak showed a systematic shift to a slightly higher mass, consistent with a population containing a reduced disulfide bond. Modification with MMTS revealed about $28 \%$ of the one-metal SOD1 contained a reduced disulfide bond (not shown). In the presence of diamide, the contribution of the higher mass component was absent, consistent with oxidation of the disulfide bond.

The sensitivity of the ZipTip assay allowed isotopicallyresolved SOD1 mass spectra to be measured from 400 motor neurons captured from frozen sections of SOD1transgenic spinal cord using a Zeiss (Jena, Germany)
PALM MicroBeam IV laser capture microdissection system (Figure 6).

\section{Conclusions}

The accuracy of isotopically-resolved protein mass data offers a means to measure overlapping non-covalent protein post-translational modifications. The method is exquisitely sensitive, allowing us to measure metal contents of SOD1 from even a few hundred cells collected by laser capture microdissection. The quantification of post-translational modifications was also facilitated by the ZipTip, which allows bound proteins to be probed with classic biochemical protein modifying reagents to examine thiol oxidation and could be extended to measure other modifications. The thiol modification assays have proven to be extremely useful in assessing the correct formation of disulfides in other recombinant proteins as large as $25-30 \mathrm{kDa}$.

\section{Acknowledgments}

The authors are grateful to Rockie Yarwood of the Stable Isotope Research Unit for ${ }^{13} \mathrm{C}$ measurements. They acknowledge the Environmental Health Sciences Center Mass Spectrometry Core Facility for infrastructure support. This work was supported by the Amyotrophic Lateral Sclerosis Association, the National Institute for Environmental Health Sciences (NIEHS P30ES000210), the National Institutes of Neurological Disorders and Stroke (NINDS R01NS058628A), and the National Center for Complementary and Alternative Medicine (NCCAM P01AT002034).

\section{References}

1. Marshall, A.G., Hendrickson, C.L., Shi, S.D.: Scaling MS plateaus with high-resolution FT-ICRMS. Anal. Chem. 74(9), 252A-259A (2002)

2. Beavis, R.C.: Chemical mass of carbon in proteins. Anal. Chem. 65(4), 496-497 (1993)

3. Senko, M.W., Beu, S.C., McLafferty, F.W.: Determination of monoisotopic masses and ion populations for large biomolecules from resolved isotopic distributions. J. Am. Soc. Mass Spectrom. 6(4), 229233 (1995)

4. Rockwood, A.L., VanOrden, S.L., Smith, R.D.: Rapid calculation of isotope distributions. Anal. Chem. 67(15), 2699-2704 (1995)

5. Rockwood, A.L., VanOrden, S.L., Smith, R.D.: Ultrahigh resolution isotope distribution calculations. Rapid Commun. Mass Spectrom. 10 (1), 54-59 (1996)

6. He, F., Hendrickson, C.L., Marshall, A.G.: Unequivocal determination of metal atom oxidation state in naked heme proteins: Fe(III)myoglobin, $\mathrm{Fe}(\mathrm{III})$ cytochrome $c$, Fe(III)cytochrome b5, and $\mathrm{Fe}$ (III)cytochrome b5 L47R. J. Am. Soc. Mass Spectrom. 11(2), 120-126 (2000)

7. Green, M.K., Vestling, M.M., Johnston, M.V., Larsen, B.S.: Distinguishing small molecular mass differences of proteins by mass spectrometry. Anal. Biochem. 260(2), 204-211 (1998)

8. Rhoads, T.W., Lopez, N.I., Zollinger, D.R., Morré, J.T., Arbogast, B.L., Maier, C.S., Denoyer, L., Beckman, J.S.: Measuring copper and zinc superoxide dismutase from spinal cord tissue using electrospray mass spectrometry. Anal. Biochem. 415(1), 52-58 (2011)

9. Estevez, A.G., Crow, J.P., Sampson, J.B., Reiter, C., Zhuang, Y., Richardson, G.J., Tarpey, M.M., Barbeito, L., Beckman, J.S.: Induction of nitric oxide-dependent apoptosis in motor neurons by zinc-deficient superoxide dismutase. Science 286(5449), 2498-2500 (1999) 
10. Hornberg, A., Logan, D.T., Marklund, S.L., Oliveberg, M.: The coupling between disulphide status, metallation and dimer interface strength in $\mathrm{Cu} / \mathrm{Zn}$ superoxide dismutase. J. Mol. Biol. 365(2), 333-342 (2007)

11. Jonsson, P.A., Graffmo, K.S., Andersen, P.M., Brannstrom, T., Lindberg, M., Oliveberg, M., Marklund, S.L.: Disulphide-reduced superoxide dismutase- 1 in $\mathrm{CNS}$ of transgenic amyotrophic lateral sclerosis models. Brain 129(Pt 2), 451-464 (2006)

12. Carroll, M.C., Outten, C.E., Proescher, J.B., Rosenfeld, L., Watson, W.H., Whitson, L.J., Hart, P.J., Jensen, L.T., Cizewski Culotta, V.: The effects of glutaredoxin and copper activation pathways on the disulfide and stability of $\mathrm{Cu}, \mathrm{Zn}$ superoxide dismutase. J. Biol. Chem. 281(39), 28648-28656 (2006)

13. Furukawa, Y., Fu, R., Deng, H.X., Siddique, T., O'Halloran, T.V.: Disulfide cross-linked protein represents a significant fraction of als-associated $\mathrm{Cu}, \mathrm{Zn}$-superoxide dismutase aggregates in spinal cords of model mice. Proc. Natl. Acad. Sci. U. S. A. 103(18), 7147153 (2006)

14. Jonsson, P.A., Ernhill, K., Andersen, P.M., Bergemalm, D., Brannstrom, T., Gredal, O., Nilsson, P., Marklund, S.L.: Minute quantities of misfolded mutant superoxide dismutase-1 cause amyotrophic lateral sclerosis. Brain 127(Pt 1), 73-88 (2004)

15. Karch, C.M., Prudencio, M., Winkler, D.D., Hart, P.J., Borchelt, D.R.: Role of mutant SOD1 disulfide oxidation and aggregation in the pathogenesis of familial ALS. Proc. Natl. Acad. Sci. U. S. A. 106(19), 7774-7779 (2009)

16. Hayward, L.J., Rodriguez, J.A., Kim, J.W., Tiwari, A., Goto, J.J., Cabelli, D.E., Valentine, J.S., Brown Jr., R.H.: Decreased metallation and activity in subsets of mutant superoxide dismutases associated with familial amyotrophic lateral sclerosis. J. Biol. Chem. 277(18), 1592315931 (2002)
17. McCord, J.M., Fridovich, I.: Superoxide dismutase: An enzymic function for erythrocuprein (hemocuprein). J. Biol. Chem. 244(22), 6049-6055 (1969)

18. O'Leary, M.H.: Carbon isotopes in photosynthesis. BioScience 38(5), 328-336 (1988)

19. Mann, M., Meng, C.K., Fenn, J.B.: Interpreting mass-spectra of multiply charged ions. Anal. Chem. 61(15), 1702-1708 (1989)

20. Ledford, E.B., Rempel, D.L., Gross, M.L.: Space-charge effects in Fourier-transform mass-spectrometry. II. Mass calibration. Anal. Chem. 56(14), 2744-2748 (1984)

21. Ledford, E.B., Rempel, D.L., Gross, M.L.: Space-charge effects in Fourier-transform mass-spectrometry. I. Electrons. Int. J. Mass Spectrom. 55(2), 143-154 (1984)

22. Uechi, G.T., Dunbar, R.C.: Space-charge effects on relative peak heights in Fourier transform-ion cyclotron-resonance spectra. J. Am. Soc. Mass Spectrom. 3(7), 734-741 (1992)

23. O'Leary, M.H., Rife, J.E., Slater, J.D.: Kinetic and isotope effect studies of maize phosphoenolpyruvate carboxylase. Biochemistry-US 20(25), 7308-7314 (1981)

24. Farquhar, G.D., Ehleringer, J.R., Hubick, K.T.: Carbon isotope discrimination and photosynthesis. Annu. Rev. Plant Physiol. 40, 503537 (1989)

25. Deniro, M.J., Epstein, S.: Influence of diet on distribution of carbon isotopes in animals. Geochim. Cosmochim. Acta 42(5), 495-506 (1978)

26. Wu, Q.Y. VanOrden, S., Cheng, X.H., Bakhtiar, R., Smith, R.D.: Characterization of cytochrome $c$ variants with high-resolution FTICR mass-spectrometry - correlation of fragmentation and structure. Anal. Chem. 67(14), 2498-2509 (1995)

27. Karala, A.R., Ruddock, L.W.: Does S-methyl methanethiosulfonate trap the thiol-disulfide state of proteins? Antioxid. Redox. Signal. 9(4), 527-531 (2007) 